\title{
JAVA based software for analizing pseudo-Weissenberg imaging plate
}

\author{
S.Weber and A.Yamamoto, NIRIM, Namiki 1-1, Tsukuba, Ibaraki 305, Japan
}

We present a preliminary report on the status of our work concerning a software package called JWEISS which is applicable to crystals and quasicrystals and serves to extract reflection intensities from pseudo-Weissenberg imaging plate data. This visually oriented software is written in the JAVA programming language which allows an easy creation of the graphical user interface (GUI). At present CPU-intensive routines are however linked in the form of native C code, since JAVA by its nature as an interpreted language is still too slow for heavy calculations and disc access. The aim of this software is to extract the integrated $\mathrm{x}$-ray intensities for the quasicrystal structure analysis.

In the pseudo-Weissenberg technique no layer screen is used. Therefore reflections belonging to several layers are recorded on one IP but the rotation angle is limited. By taking a series of images one can therefore record several complete layers including satellite reflections. Especially in the case of quasicrystals this will drastically reduce the time needed for a data collection, since several thousands of reflections can be recorded simultanously. During the development of the software we use example imaging plate data for triclinic $\mathrm{Pb}_{11} \mathrm{Ge}_{3} \mathrm{O}_{17}(\mathrm{a}=22.259 \AA$, $\mathrm{b}=19.910 \AA$, $\mathrm{c}=7.361 \AA$, $\alpha=97.59^{\circ}, \beta=95.15^{\circ}, \gamma=92.35^{\circ}$ ) provided by K.Kato (NIRIM) ${ }^{[1]}$.

For displaying and manipulating the data within JWEISS complete reciprocal layers are reconstructed by merging strips from the individual IP files and converting them to GIF images. Furthermore precession photo images are created by a coordinate transformation. These precession photo images serve the determination of some starting parameters, such as initial rotation angle and lattice parameters. Then a Weissenberg simulation is calculated and overlayed on the Weissenberg photo in order to index the peaks. The assigned peaks are then fitted by a profile function in order to obtain the integrated intensities.

\section{References}

[1] K.Kato, K.Hirota, Y.Kanke, A.Sato, K.Ohsumi, T.Takase, M.Uchida, O.Jarchow, K.Friese, G.Adiwidjaja: Zeit.f.Krist. 210 (1995), 188-194

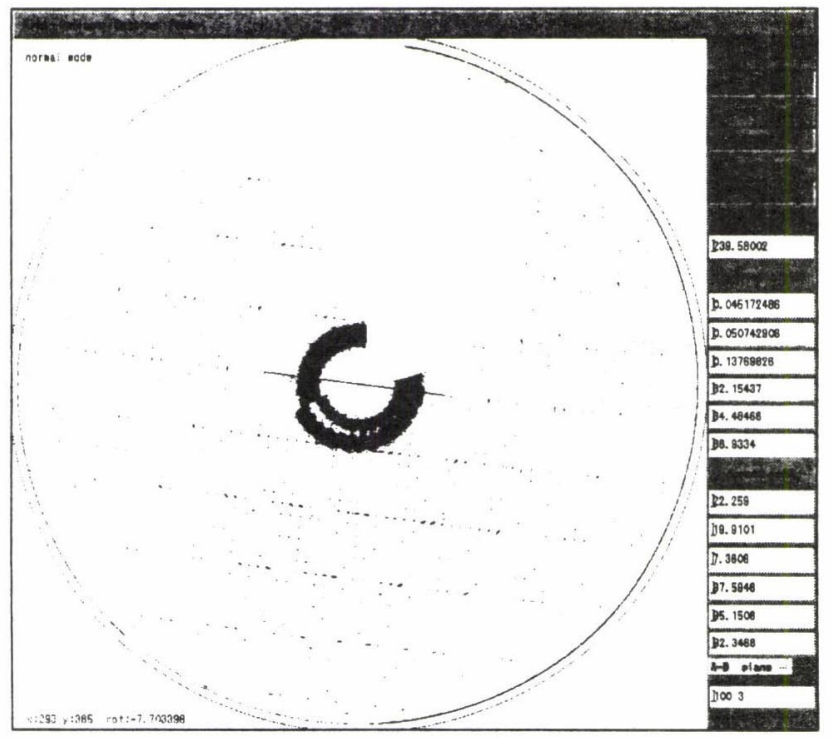

Fig. Screen-shot of a window in precession mode showing the (hk0)-reciprocal plane. 